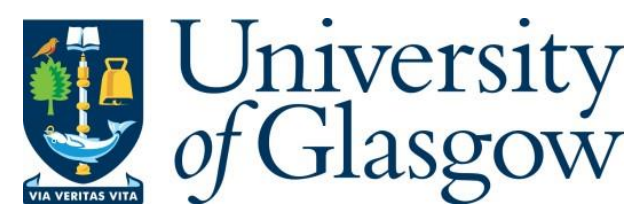

Zhou, B., Le Kernec, J., Yang, S., Fioranelli, F., Romain, O. and Zhao, Z. (2021) Interferometric Radar for Activity Recognition and Benchmarking in Different Radar Geometries. In: IET International Radar Conference 2020, Chongqing City, China, 4-6 Nov 2020, pp. 1515-1520. ISBN 9781839535406.

There may be differences between this version and the published version. You are advised to consult the publisher's version if you wish to cite from it.

http://eprints.gla.ac.uk/223728/

Deposited on: 1 October 2020

Enlighten - Research publications by members of the University of Glasgow http://eprints.gla.ac.uk 


\title{
Interferometric radar for activity recognition and benchmarking in different radar geometries
}

\author{
Boyu Zhou', Julien Le Kernec ${ }^{2,3,4}$, Shufan Yang ${ }^{3}$, Francesco Fioranelli, ${ }^{5}$ Olivier Romain ${ }^{4}$, Zhiqin $^{2}$ \\ Zhao $^{2}$ \\ ${ }^{1}$ Department of Computer Science, the University of Hong Kong, Hong Kong, China \\ ${ }^{2}$ School of Information and Communication, University of Electronic Science and Technology of China, Chengdu, China \\ ${ }^{3}$ James Watt School of Engineering, University of Glasgow, Glasgow, UK \\ ${ }^{4}$ ETIS - Signal and Information Processing lab, University Cergy-Pontoise, Cergy, France \\ ${ }^{5}$ MS3-Microwave Sensing Signals and Systems, TU Delft, Delft, The Netherlands \\ *Julien.lekernec@glasgow.ac.uk
}

Keywords: CLASSIFICATION, HUMAN ACTIVITY RECOGNITION, HUMAN MICRO-DOPPLER, MACHINE LEARNING.

\begin{abstract}
Radar micro-Doppler signatures have been proposed for human activity classification for surveillance and ambient assisted living in healthcare-related applications. A known issue is the performance reduction when the target is moving tangentially to the line-of-sight of the radar. Multiple techniques have been proposed to address this, such as multistatic radar and to some extent, interferometric radar. A simulator is presented to generate synthetic data representative of 8 different radar systems (including configurations as monostatic, multistatic, and interferometric) to quantify classification performances as a function of aspect angles and deployment geometries. This simulator allows an unbiased performance evaluation of the different radar systems. 6 human activities are considered with signatures originating from motion-captured data of 14 different subjects. The results show that interferometric radar data with fusion outperforms the other methods with over $97.6 \%$ accuracy consistently across all aspect angles, as well as the potential for simplified indoor deployment.
\end{abstract}

\section{Introduction}

Radar signatures, in particular, micro-Doppler $(\mathrm{mD})$ signatures $(\mathrm{mDs})$, have attracted significant interests for human activity classification for security, healthcare, and assisted living applications [1].

An issue for classification based on $\mathrm{mDs}$ is the performance reduction for targets' trajectories tangential to the radar line of sight, as the $\mathrm{mD}$ frequency shifts are reduced, and it is challenging to extract informative features from the data. For example, Tahmoush [2] showed that micro-Doppler classification performance dropped to $40 \%$ at high aspect angles, and references [3-5] analysed the classification performance and limitations due to the aspect angle. When the target is not walking or moving along the radial direction, depending on the aspect angle, the salient features for classification may change, and the accuracy of classification reduces as the target velocity gets closer to the tangential direction.
As monostatic (MN) radar can only observe well the radial component of the $\mathrm{mD}$ signal, multiple cooperating radar sensors have been suggested to enhance the classification of $\mathrm{mDs}$. This provides additional information from multistatic (MS) perspectives, at the price of increased system complexity to synchronize the different nodes $[6,7]$ separated by a baseline (the distance between nodes, e.g. transmitter (Tx) to receiver (Rx) in the bistatic case). An early implementation of simulated MS radar mDs from the Boulic model [8] for walk is presented in [9]. It shows how the fusion of the mDs of several nodes together may improve the quality and clarity of the $\mathrm{mDs}$ in comparison to only one node considering aspect angles at 0,30 and $75^{\circ}$ and different signal-to-noise ratios.

Interferometric (IFM) information has also been suggested as an alternative/complementary technique. Nanzer, in [10], presented an analysis of the angular velocity measurement of a person who is walking via a millimetrewave correlation interferometer, which also covered the IFM measurement theory of angular velocity, and the frequency response simulations of a walking human participant.

The IFM channel provided information about the target angular velocity. This IFM signature is more pronounced as the baseline between the antennas is increasing. They showed that as the trajectory moved from a completely radial motion to completely tangential motion, the Doppler frequency shift decreased. In contrast, the IFM frequency shift increased for the walking action. Hence, these 2 detection modes can represent complementary measurements, improving the ability to measure the motion of randomly moving objects.

The research community has extensively focused on $\mathrm{mDs}$ for human radar classification. Still, this domain has shown limitations to distinguish between activities showing similar radial acceleration with respect to the radar, a.k.a. confusers. MS radar has been used to enhance classification accuracy with confusers and to tackle aspect angle issues, but the IFM sensing modalities has seldom been used in the literature.

In this paper, an IFM radar geometry is proposed that demonstrates robustness with respect to the aspect angle for indoor human activity recognition applications. The focus of this work is on generating synthetic $\mathrm{mDs}$ and, by association, IFM data. Several techniques have been used on $\mathrm{mDs}$ as it represents the majority of contributions on human activity recognition with radar to date. The classification techniques 
include Bi-LSTM [11], LSTM [12], GRU [13], SAE, CAE [14], CNN [15], MFCC/FWCC [16] leading to increasingly better performances. A shallow CNN network (LeNet-5 [17]) has been selected here to perform the transfer learning from optical recognition to radar activity recognition in this work.
The paper is organized as follows. Section 2 describes the methodology to establish the simulation. Section 3 compares the results between the 8 radar systems. Finally, conclusions are given in Section 4.
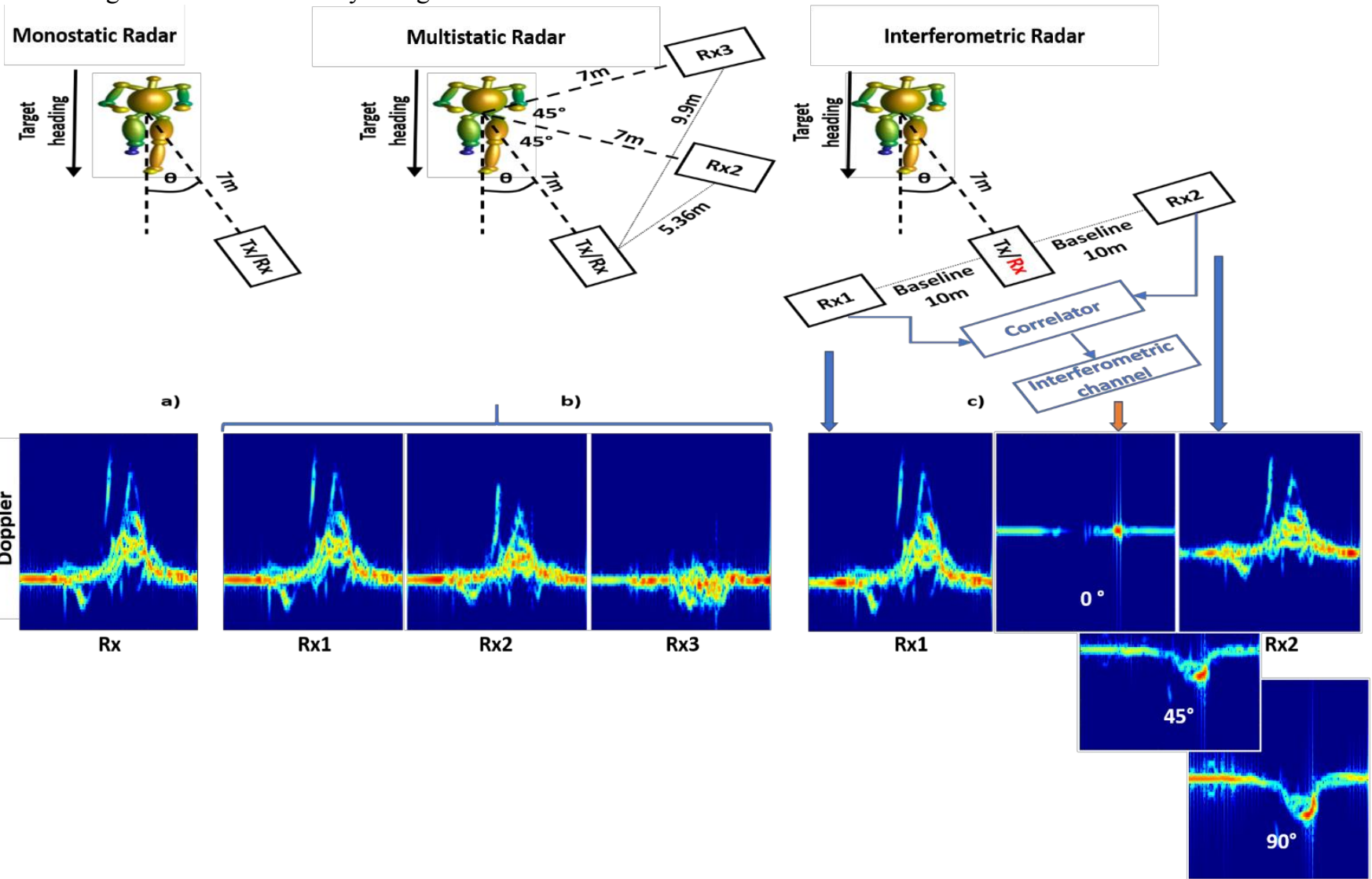

Fig. 1. The simulation geometry for (a) MN radar system, (b) MS radar system - circular configuration with one transceiver and two bistatic receivers, and (c) IFM radar system (black and blue) \& MS radar-in-line configuration (black and red).

\section{Methodology of the simulator}

A framework to simulate and compare performances of 8 radar systems, was developed. This included a MN radar (Fig. 1a): relying on $\mathrm{mDs}$ from a single $\mathrm{MN}$ radar node; a MS radar (Fig. 1b): using 3 separate nodes, whose results are fused for classification purposes using majority voting; a MS radar (Fig. 1c black and red): using 3 separate nodes with baselines of 2 , $5 \& 10 \mathrm{~m}$, whose results are fused for classification purposes using a majority voting approacht; and finally an IFM radar (Fig. 1c black and blue): using 2 Rxs with baselines of 2, 5, $\& 10 \mathrm{~m}$, whose results are fused with the IFM channel for classification using majority voting.

Fig. 2 shows the Doppler shifts for a carrier at $9.8 \mathrm{GHz}$ for a target at $1 \mathrm{~m} / \mathrm{s}$ at the centre of the scene at varying aspect angles based on the theory in [7, 18]. The circular configuration was chosen at 0,45 , and $90^{\circ}$ as it offered more diversity in Doppler shifts for a more robust classification compared to narrower bistatic angles with a Tx placement on the side as in [9]. The in-line configuration is inspired by [19]. Fig. 2a shows the most extensive variation in Doppler shifts with a 10m-baseline. As for the IFM channel, the configuration is based on [20].

The performance comparison is based on the accuracy of classification for 6 human motions where the aspect angle $\theta$ between the target heading and the radar line of sight changes from $0^{\circ}$ to $90^{\circ}$ with $5^{\circ}$ per step in rotation. The details of the geometry of the different radar setups are shown in Fig. 1. It is important to note that the target may be translating, and the aspect angle does not remain constant. Instead, the heading of the target is considered to define the aspect angle.

The 6 classes of motions considered include (I) walking; (II) forward jumping; (III) kicking; (IV) sitting and standing; (V) running; (VI) walking on uneven terrain. These data originate from the Carnegie Mellon Motion capture (MoCap) database [15] or the HDM05 MoCap database [21]. Motion data in ASF/AMC format were used since this kind of skeletonbased data can comprise an explicit skeleton structure and also ensure that the bone lengths will be constant in the movement [15]. Motion data for head, torso, pelvis, legs, feet, arms, and hands were used to simulate radar returns.

The motion data was captured at $120 \mathrm{~Hz}$ in the database [15]. To simulate the Doppler frequency shift without aliasing, the sampling frequency was upsampled to $2 \mathrm{kHz}$ before the simulation. The animation of human movement code was modified from [22], in particular, to generate MS and IFM signals and signatures.

Figure 3 shows a flow graph of the overall process for generation of synthetic radar signatures. For simplicity, the simulator assumed no free space losses, a noise-free environment, and no specific beam pattern. The total radar 
return is a sum of backscattered signals from separate body parts. To get accurate radar returns, several parameters about the radar system should be set according to the specific scenarios, including the radar location, the bandwidth of the transmitted (Tx) signal (here equal to $400 \mathrm{MHz}($, and the carrier frequency (here $9.8 \mathrm{GHz}$ ). In simulating $\mathrm{mDs}$, the ranges from the $\mathrm{Tx} / \mathrm{Rx}$ to the target are represented by (1).

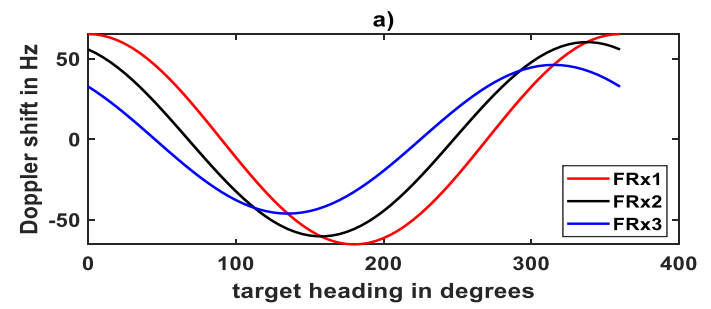

b)

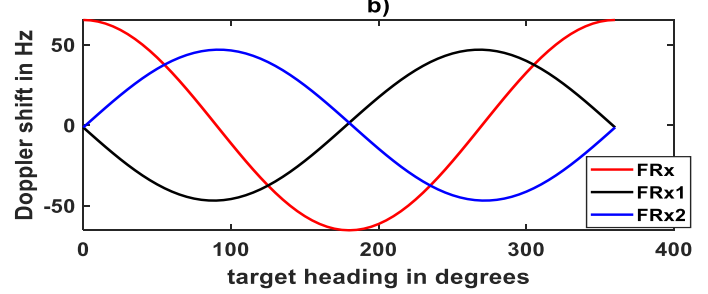

Fig. 2. The expected Doppler shifts for a) the circular MS configuration with nodes at 0,45 , and $90^{\circ}$; b) the in-line configuration with a $10 \mathrm{~m}$ baseline (Rx1/Rx2 have the same Doppler shifts in the IFM case) for a carrier frequency of 9. $8 \mathrm{GHz}$.

$$
r_{T}=r_{t}+v t, \quad r_{R}=r_{r}+v t
$$

$r_{t}$ and $r_{r}$ denote the initial ranges from the Tx antenna and the $\mathrm{Rx}$ antenna to the target respectively; $r_{T}$ and $r_{R}$ denote the ranges as a function of time $t ; v$ denotes the relative radial velocity of the moving object to the radar. The total phase change between the Tx signal and the scattered signal is proportional to the total length of propagation, which can be calculated by (2).

$$
\Delta \phi=2 \pi \frac{r_{T}+r_{R}}{\lambda_{c}}
$$

where $\lambda_{c}=c / f_{c}$ denotes the $\mathrm{Tx}$ signal wavelength, $c$ denotes the speed of light in $\mathrm{m} / \mathrm{s}$, and $f_{c}$ is the carrier frequency. When the Tx and the Rx are static, the Doppler shift $f_{d}$ can be calculated by (3).

$$
f_{d}=\frac{1}{2 \pi} \frac{d}{d t} \Delta \phi=\frac{2 v}{\lambda_{c}}
$$

where the observed frequency is proportional to the relative radial velocity. The complex Doppler signal at the $\mathrm{Rx}$ can be represented by (4)

$$
S(t)=A e^{-j 2 \pi f_{c}\left(\frac{2 r(t)}{c}\right)}
$$

where $A$ denotes the radar cross-section (RCS), $f_{c}$ is the frequency of the Tx signal, and $r(t)$ denotes the distance from the radar to the target [22].

The simulation represents the 2 received $(\mathrm{Rx})$ signals of the IFM radar system by (5).

$$
S_{1}(t)=A_{1} e^{j 2 \pi f_{r} t}, S_{2}(t)=A_{2} e^{j 2 \pi f_{r}(t-\tau)}
$$

where $\tau$ denotes the time delay of signal reception between the $2 \mathrm{Rx}$ signals [23], $A_{1} \& A_{2}$ denote the target RCS viewed from $\mathrm{Rx} 1 \& 2$, and $f_{r}$ is the $\mathrm{Rx}$ frequency. For the IFM measurement, the time delay can be calculated by (6).

$$
\tau=\frac{D}{c} \sin (\theta)=\frac{D}{c} \sin (\omega t)
$$

where $\omega$ is the angular velocity of the target, $\theta$ denotes the angle off the broadside, $D$ is the baseline between the 2 Rxs, and $c$ is the speed of the electromagnetic signal. The IFM response after correlator is the product and integration of the 2 Rx signals as shown in (7) [23].

$$
S_{i}(t)=A_{1} A_{2} e^{j 2 \pi f_{r} \tau}=A_{1} A_{2} e^{j 2 \pi f_{r} \frac{D}{c} \sin (\omega t)}
$$

where $f_{r}$ is the frequency of the Rx signal.

The observed IFM frequency can be calculated by (8).

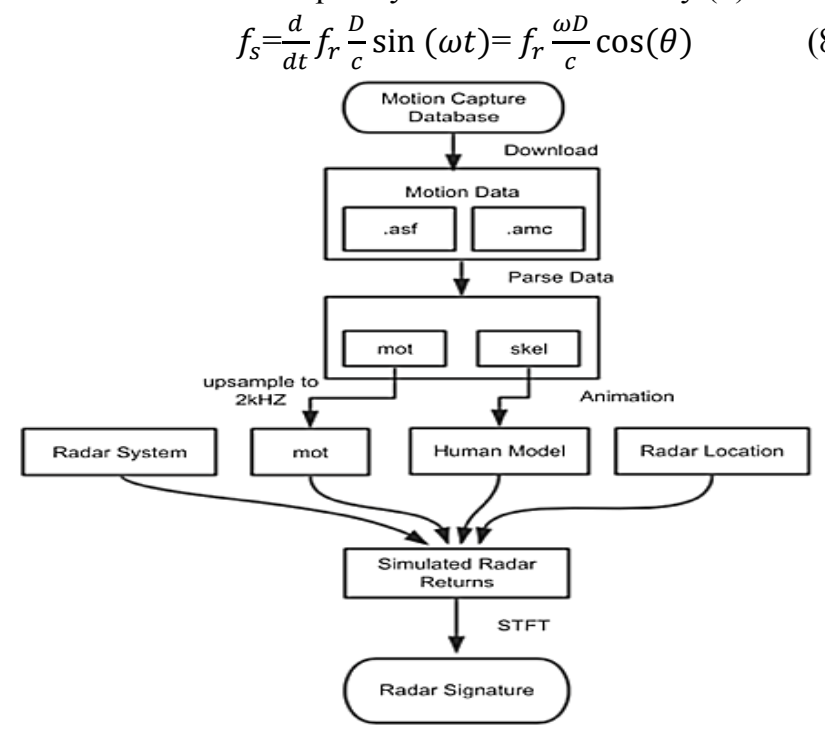

Fig. 3. Flow graph of the radar signature simulator algorithm based on motion-captured data

In the simulation, the value of the RCS is used as the amplitude of the complex response signal. If the Rx and the Tx are not located at the same place, the RCS should be calculated by its bistatic expression. The resulting IFM and Doppler responses are both processed in the time-frequency domain using Short Time Fourier Transform (STFT). The STFT separates the time-varying signal into shorter segments using overlapped Gaussian windows with a length of 256 samples (128 $\mathrm{ms}$ at $2 \mathrm{kHz}$ pulse repetition period) and processes each data window using Fourier transform to calculate the frequency components.

A total of 88 different motion files performed by 14 subjects from CMU database were simulated to generate the training/testing datasets. These were used to generate the frequency responses at different aspect angles. It was not possible to have all 6 actions all the time from the same subject due to the limitations of this dataset. Samples from every trial data were divided as 1-second long snapshots to increase the size of the dataset. Every class has 80 samples, thus 480 in total. 360 samples $(75 \%)$ are chosen as the training data, and the classification algorithm will test the other 120 samples $(25 \%)$ in every scenario (Table 1$)$. The test samples for different classification methods were simulated from the same motion data files to ensure a fair comparison. Convolutional Neural Networks (CNNs) use mDs as input for classification and extract features automatically. The MN radar relies on one $\mathrm{mDs}$, processed by a single CNN. The IFM 
radar system implemented majority voting [24] as a decisionlevel fusion mechanism on the output labels from $2 \mathrm{mDs}$, and one IFM frequency response. Therefore, it needed 3 separate CNNs. The MS radar system also utilized the same fusion mechanism from $3 \mathrm{mDs}$ from different radar nodes, hence it also needed 3 CNNs. In total, in every tested scenario, one $\mathrm{CNN}$ was trained per channel using the corresponding frequency responses and $\mathrm{mDs}$.

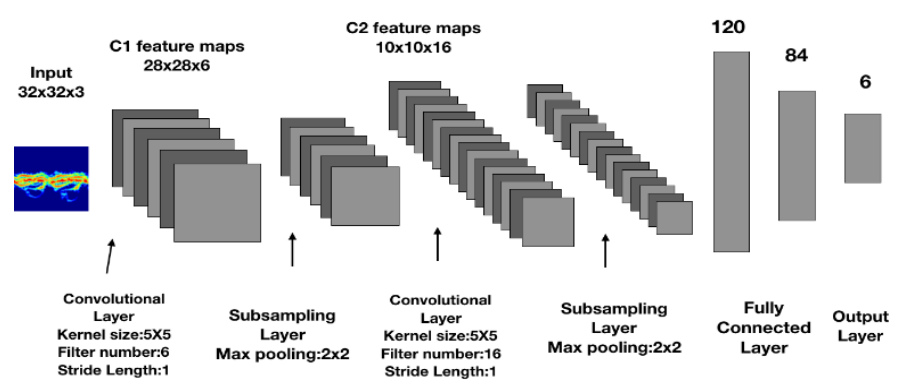

Fig. 4. Structure of CNN used in this article as a classifier

Table 1 Summary of motion data samples from CMU

\begin{tabular}{|l|l|c|c|c|}
\hline Actions & Number of subjects & Number of files & Training set & Testing set \\
\hline I walking & 3(subject 02,07,08) & 18 & 60 & 20 \\
\hline II forward jumping & $\begin{array}{l}5 \text { (subject } \\
13,16,83,91,105)\end{array}$ & 23 & 60 & 20 \\
\hline III kicking & 1 (subject 144) & 4 & 60 & 20 \\
\hline IV sitting and standing & 2 (subject 13,13) & 9 & 60 & 20 \\
\hline V running & 2 (subject 09,35) & 21 & 60 & 20 \\
\hline $\begin{array}{l}\text { VI walking on uneven } \\
\text { terrain }\end{array}$ & ( (subject 36) & 13 & 60 & 20 \\
\hline
\end{tabular}

Table 2 Hyperparameters for CNN

\begin{tabular}{|l|c|}
\hline Hyperparameters & Value \\
\hline Learning rate & $0.012 \sim 0.013$ \\
\hline Mini Batch size & 24 \\
\hline L2 Regularization ratio & 0.0005 \\
\hline Dropout rate & 0.5 \\
\hline Solver & Stochastic Gradient Descent \\
\hline Momentum & 0.9 \\
\hline MiniBatchSize & 16 \\
\hline Max Epochs & 150 \\
\hline
\end{tabular}

The network architecture modified from LeNet-5 [17] (Fig. 4), was adopted for all of CNNs with only small differences in the hyperparameters, as given in Table 2.

Summarising, the signatures are generated by an accurate simulation of human motion based on motion-captured data from live volunteers [15]. Thus, the kinematics reflect natural movements. The signatures are based on proven simulation methods from [22], which is then extended using analytical equations for RCS in $\mathrm{MN}$ and bistatic configurations from [25]. Lastly, the theoretical frameworks for the bistatic and IFM channels were demonstrated in [7, 10, 20, 23].

\section{Results and discussions}

\section{1. $m D$ and IFM responses examples}

For all 8 radar systems, the signatures for each of the Rxs were captured from $0^{\circ}$ to $90^{\circ}$ with $5^{\circ}$ steps in rotation. This section comments on some examples of the signatures to visualize the phenomenology better. Fig. 1 showed the $\mathrm{mDs}$ of the transceiver node from the circular MS radar system at 3 aspect angles $0^{\circ}, 45^{\circ}$, and $90^{\circ}$. It can be observed from the $\mathrm{mDs}$ that the Doppler spread and mean decrease as the aspect angle in rotation increases from $0^{\circ}$ to $90^{\circ}$. At $90^{\circ}$, the action is barely distinguishable.

Fig. 1 also depicted the IFM response obtained at different aspect angles for a fixed baseline of $10 \mathrm{~m}$. The IFM channel increases in amplitude as the aspect angle increases from $0^{\circ}$ to $90^{\circ}$. Furthermore, the greater the baseline, the larger the amplitude registered is expected to be, as the aspect angle increases.

Note, that there are spikes in the responses; those are simulation artefacts caused by the processing by segments known as edge effects [22], and they occur at the junction between 2 segments of processed data.

\subsection{Comparison of different radar systems}

These 8 radar geometries were compared in classification accuracy for the scenarios where the aspect angle changes from $0^{\circ}$ to $90^{\circ}$ with $5^{\circ}$ steps. In every chosen aspect angle, every network associated with each radar channel was repeatedly trained ten times through a cross-validation process, and then their decisions were fused. The final accuracy, as shown in Fig. 5, is based on the average classification results for $\mathrm{MN}$ (1 network), circular and in-line MS (3 networks and decision-level fusion), and IFM (3 networks and decision-level fusion) configurations.

The MS radar (in-line or circular) performs best from $0^{\circ}$ to $35^{\circ}$ with up to $1.15 \%$ improvement in accuracy. However past $35^{\circ}$, the performances of the circular MS radar drop under the performance of the IFM and in-line MS systems. The method relying only on the single $\mathrm{mD}$ signature from the $\mathrm{MN}$ radar system shows a downward trend in the performance, and it degrades severely where the aspect angle is larger than $65^{\circ}$, as expected. The average accuracy is only $80.92 \%$ at $90^{\circ}$. Although the circular MS radar performs better than $\mathrm{MN}$, its accuracy drops under $95 \%$ after $45^{\circ}$ and even lower between 80 to $90^{\circ}$ aspect angles. It can be observed that the IFM radar maintains over $97.58 \%$ accuracy for all aspect angles ranging from $0^{\circ}$ to $90^{\circ}$, whereas the inline MS maintains 94\% accuracy. The in-line maintains consistent performances up to $60^{\circ}$ with the IFM radar. The IFM radar accuracy improves by $+2.4 \%$ at $45^{\circ}$ to $+16.6 \%$ at $90^{\circ}$ compared to $\mathrm{MN}$ radar. The accuracy up to $40^{\circ}$ is comparable for the MS and IFM systems. The IFM system outperforms the in-line MS radar for larger aspect angles between 75 and $90^{\circ}$.

To further investigate the robustness of the proposed methods, the baseline between the Txs and the Rxs of the IFM radar was also set to 2 and $5 \mathrm{~m}$, respectively. The classification results are shown in Fig. 5. The radar system with a $5 \mathrm{~m}$ baseline has similar performances with the $10 \mathrm{~m}$ baseline, with only a minor performance degradation ranging from $[0.1$ to $1.5 \%$ ] for the IFM radar. The average accuracy with a $5 \mathrm{~m}$ baseline in every scenario fluctuates around $98 \%$, and its minimum value is $97.08 \%$. Below $55^{\circ}$ aspect angle, the IFM radar with a $2 \mathrm{~m}$ baseline performs similarly to the $10 \mathrm{~m}$ baseline radar, but the performances decline to $91.58 \%$ at $90^{\circ}$. Additionally, the $2 \mathrm{~m}$-baseline IFM system has better performances than the MN, 2m-baseline in-line MS, and circular MS radar systems. The in-line IFM radar system, however, is not performing well with a reduction in the baseline with performances dropping severely after $55^{\circ}$ to $94 \%$ and $\sim 91 \%$ at $90^{\circ}$ with $5 \mathrm{~m}$ baseline. Furthermore, the stability, 
up to $55^{\circ}$ worsens and fluctuates between 97 and $99 \%$. The degradations are worse with $2 \mathrm{~m}$ baseline.

\section{Conclusion}

In this paper, 8 radar systems, namely MN, MS (circular \& in-line), and IFM for 6 classes of motions were simulated and compared in scenarios where the aspect angle to the target changes from $0^{\circ}$ to $90^{\circ}$. A total of 88 different motion data files performed by 14 human subjects from CMU were simulated to generate corresponding $\mathrm{mDs}$ and corresponding interferograms. In every scenario, one $\mathrm{CNN}$ was trained per channel to perform the tasks of classification and comparison. From the simulation results given in Section 3, the IFM radar with suitable baseline and fusion of the $\mathrm{mD}$ signatures and the IFM frequency responses has more consistent capabilities over the other systems to discriminate between the different activities, throughout the whole range $0^{\circ}$ to $90^{\circ}$ in aspect angle. The joint use of IFM frequency response and $\mathrm{mD}$ signatures yield an accuracy of over $97.58 \%$ in all scenarios with a $10 \mathrm{~m}$ baseline and $97.08 \%$ with $5 \mathrm{~m}$. Even with a reduction in the baseline, the IFM radar maintains good performances.
In contrast, the performance degradation for the in-line MS radar would not be acceptable for operational deployment considering indoor applications. Additionally, for the IFM radar, the higher the carrier frequency, the smaller the baseline has to be to benefit from the same performances. With the advent of millimetre-wave technologies, a similar level of performances will be available with a much smaller form factor for indoor scenarios, whereas in-line MS radar would require a significant baseline to maintain good performances and may not be suitable for indoor environments.

Future work will look at feature level fusion for the implementation of classification to reduce the computational load, and lightweight implementation of the networks to reduce their size and time for training and inference.

\section{Acknowledgment}

The authors would like to thank the British Council 515095884 and Campus France 44764WK - PHC Alliance France-UK for their financial support.

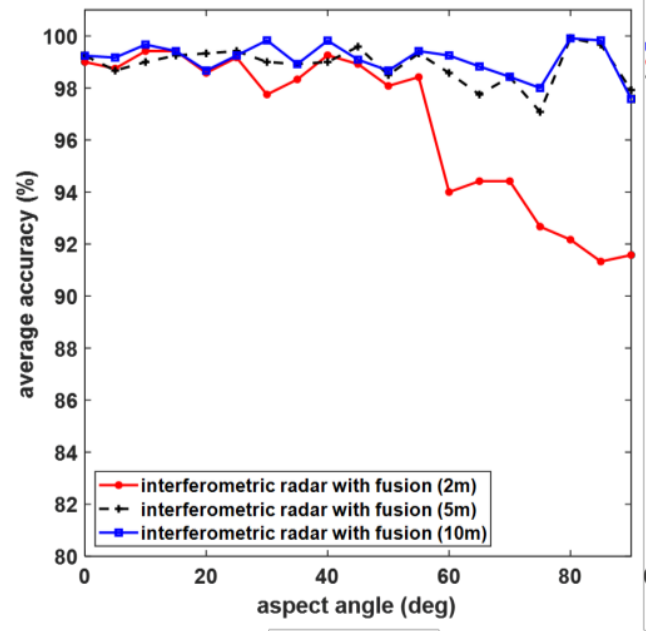

a)

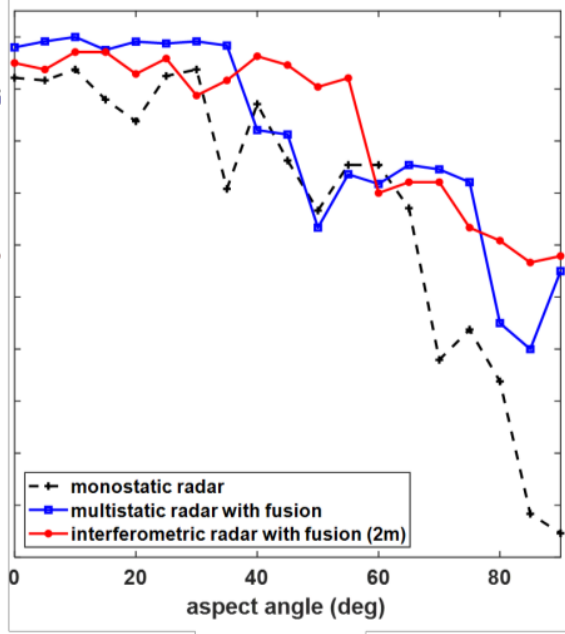

b)

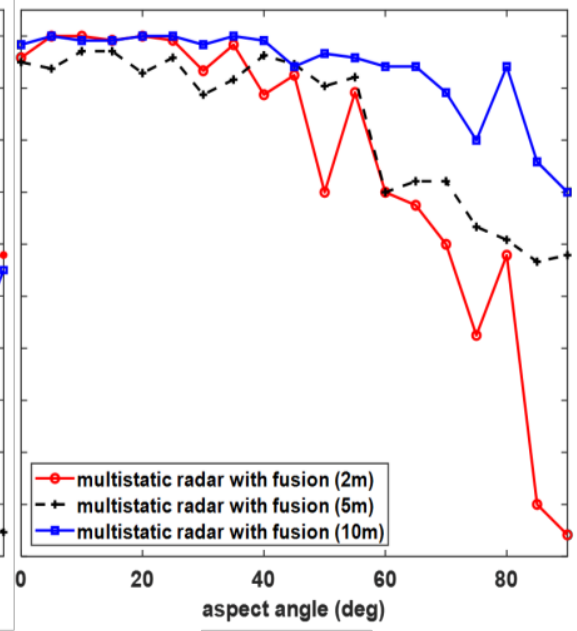

c)

Fig. 5. Comparison of results a) Classification results of IFM radar systems with $2 \mathrm{~m}$, 5m, $10 \mathrm{~m}$ baseline respectively; b) comparison of IFM radar with $2 m$ baseline with MN and MS radar (circular); c) comparison of MS radar (in-line) with $2 m, 5 m$ and $10 \mathrm{~m}$ baseline .

\section{References}

[1] J. L. Kernec et al., "Radar Signal Processing for Sensing in Assisted Living: The challenges associated with realtime implementation of emerging algorithms," IEEE Signal Processing Magazine, vol. 36, no. 4, pp. 29-41, 2019, doi: 10.1109/MSP.2019.2903715.

[2] D. Tahmoush and J. Silvious, "Radar micro-doppler for long range front-view gait recognition," in 2009 IEEE 3rd International Conference on Biometrics: Theory, Applications, and Systems, 28-30 Sept. 2009 2009, pp. 16, doi: 10.1109/BTAS.2009.5339049.

[3] B. Tekeli, S. Z. Gurbuz, and M. Yuksel, "InformationTheoretic Feature Selection for Human Micro-Doppler Signature Classification," IEEE Transactions on Geoscience and Remote Sensing, vol. 54, no. 5, pp. 27492762, 2016, doi: 10.1109/TGRS.2015.2505409.
[4] S. Z. Gürbüz, B. Erol, B. Çağlıyan, and B. Tekeli, "Operational assessment and adaptive selection of microDoppler features," IET Radar, Sonar \& Navigation, vol. 9, no. 9, pp. 1196-1204, 2015, doi: 10.1049/ietrsn.2015.0144.

[5] Y. Kim and H. Ling, "Human Activity Classification Based on Micro-Doppler Signatures Using a Support Vector Machine," IEEE Transactions on Geoscience and Remote Sensing, vol. 47, no. 5, pp. 1328-1337, 2009, doi: 10.1109/TGRS.2009.2012849.

[6] F. Fioranelli, M. Ritchie, and H. Griffiths, "Centroid features for classification of armed/unarmed multiple personnel using multistatic human micro-Doppler," IET Radar, Sonar \& Navigation, vol. 10, no. 9, pp. 17021710, 2016.

[7] N. J. Willis, Bistatic radar. Scitech publishing Inc., 2005. 
[8] R. Boulic, N. M. Thalmann, and D. Thalmann, "A global human walking model with real-time kinematic personification," The Visual Computer, vol. 6, no. 6, pp. 344-358, 1990/11/01 1990, doi: 10.1007/bf01901021.

[9] C. Karabacak, S. Z. Gürbüz, and A. C. Gürbüz, "Radar simulation of human micro-Doppler signature from video motion capture data," in 2013 21st Signal Processing and Communications Applications Conference (SIU), 24-26 April 2013 2013, pp. 1-4, doi: 10.1109/SIU.2013.6531365.

[10] J. A. Nanzer, Interferometric measurement of the angular velocity of moving humans (SPIE Defense, Security, and Sensing). SPIE, 2012.

[11] H. Li, A. Shrestha, H. Heidari, J. L. Kernec, and F. Fioranelli, "Bi-LSTM Network for Multimodal Continuous Human Activity Recognition and Fall Detection," IEEE Sensors Journal, pp. 1-1, 2019, doi: 10.1109/JSEN.2019.2946095.

[12] S. Yang, J. L. Kernec, F. Fioranelli, and O. Romain, "Human Activities Classification in a Complex Space Using Raw Radar Data," presented at the International Radar Conference, Toulon, France, 23-27 Sept, 2019.

[13] M. Wang, G. Cui, X. Yang, and L. Kong, "Human body and limb motion recognition via stacked gated recurrent units network," IET Radar, Sonar \& Navigation, vol. 12, no. 9, pp. 1046-1051, 2018, doi: 10.1049/ietrsn.2018.5054.

[14] S. Z. Gurbuz and M. G. Amin, "Radar-Based HumanMotion Recognition With Deep Learning: Promising applications for indoor monitoring," IEEE Signal Processing Magazine, vol. 36, no. 4, pp. 16-28, 2019, doi: 10.1109/MSP.2018.2890128.

[15] Y. Lin, J. L. Kernec, S. Yang, F. Fioranelli, O. Romain, and Z. Zhao, "Human Activity Classification With Radar: Optimization and Noise Robustness With Iterative Convolutional Neural Networks Followed With Random Forests," IEEE Sensors Journal, vol. 18, no. 23, pp. 9669-9681, 2018, doi: 10.1109/JSEN.2018.2872849.

[16] M. S. Seyfioglu, B. Erol, S. Z. Gurbuz, and M. G. Amin, "DNN Transfer Learning From Diversified MicroDoppler for Motion Classification," IEEE Transactions on Aerospace and Electronic Systems, vol. 55, no. 5, pp. 2164-2180, 2019, doi: 10.1109/TAES.2018.2883847.

[17] Y. Lecun, L. Bottou, Y. Bengio, and P. Haffner, "Gradient-based learning applied to document recognition," Proceedings of the IEEE, vol. 86, no. 11, pp. 2278-2324, 1998, doi: 10.1109/5.726791.

[18] F. Fioranelli, M. Ritchie, and H. Griffiths, "Aspect angle dependence and multistatic data fusion for microDoppler classification of armed/unarmed personnel," IET Radar, Sonar \& Navigation, vol. 9, no. 9, pp. 1231-1239, 2015, doi: 10.1049/iet-rsn.2015.0058.

[19] Z. Chen, G. Li, F. Fioranelli, and H. Griffiths, "Personnel Recognition and Gait Classification Based on Multistatic Micro-Doppler Signatures Using Deep Convolutional Neural Networks," IEEE Geosci. Remote Sens. Lett., vol. 15, no. 5, pp. 669-673, 2018, doi: 10.1109/LGRS.2018.2806940.

[20] J. A. Nanzer, "Micro-motion signatures in radar angular velocity measurements," in 2016 IEEE Radar
Conference (RadarConf), 2-6 May 2016 2016, pp. 1-4, doi: 10.1109/RADAR.2016.7485234.

[21] M. Muller, T. Roder, M. Clausen, B. Eberhardt, B. Kruger, and A. Weber, "Documentation Mocap Database HDM05," Institut fur informatik II Universitat Bonn D53117 Bonn, Germany, CG-2007-2, 20072007.

[22] V. Chen, The Micro-Doppler Effect in Radar. London, Boston: Artech House, 2011.

[23] J. A. Nanzer and V. C. Chen, "Microwave interferometric and Doppler radar measurements of a UAV," in 2017 IEEE Radar Conference (RadarConf), 812 May 2017 2017, pp. 1628-1633, doi: 10.1109/RADAR.2017.7944468.

[24] H. Li, A. Shrestha, H. Heidari, J. Le Kernec, and F. Fioranelli, "A Multisensory Approach for Remote Health Monitoring of Older People," IEEE J. of Electromagn., RF and Microw. in Med. and Biol., vol. 2, no. 2, pp. 102 108, 2018, doi: 10.1109/JERM.2018.2827099.

[25] K. D. Trott, "Stationary Phase Derivation for RCS of an Ellipsoid," IEEE Antennas and Wireless Propagation Letters, vol. 6, pp. 240-243, 2007, doi: 10.1109/LAWP.2007.891521. 